LBNL-47941

\title{
Quantum non-locality in a two-slit interferometer for short-lived particles
}

\author{
Spencer R. Klein ${ }^{1}$ and Joakim Nystrand ${ }^{2}$ \\ 1 Lawrence Berkeley National Laboratory, Berkeley, CA 94720 \\ 2 Department of Physics, Lund University, Lund, Sweden
}

\begin{abstract}
We describe a new test of quantum nonlocality, using an interferometer for short-lived particles. The separation is large compared with the particle lifetimes. This interferometer is realized by vector meson production in distant heavy ion collisions. The mesons decay before waves from the two sources (ions) can overlap, so interference is only possible among the decay products. The post-decay wave function must retain amplitudes for all possible decays. The decay products are spatially separated, necessitating a non-local wave function. The interference is measurable by summing the product momenta. Alternately, the products positions could be observed, allowing new tests of the EPR paradox.
\end{abstract}

Typeset using REVT $\mathrm{EX}$ 
In 1935, Einstein, Podolsky and Rosen (EPR) showed that quantum mechanics required that wave functions can be non-local [1]. When a system is observed, the wave function collapses from one which contains amplitudes for a host of possible outcomes to smaller set of possibilities, in accord with the measurement. This collapse is instantaneous; much has been written about its superluminous nature. Most studies of the EPR paradox have tested Bell's inequality [2] using spin correlations, usually with photon pairs produced in a single reaction. These tests involve the spin correlations measured after the photons go through polarizers with a varying angle between the two polarizers. If the two-photon wave function is non-local, and collapses when one of the photons passes through a polarizer, then the correlation has a characteristic angular dependence, given by Bell's inequality. In the absence of a non-local connection, a different angular dependence is expected. Other tests have used 'pseudo-spin' like variables like CP, such as studies using the reaction $\Phi \rightarrow K^{+} K^{-}$ $[3]$.

We describe a very different system that is sensitive to the collapse of continuous variables in a wave function [4]. Short-lived particles are produced at two separated sources, with a fixed phase relationship, forming an interferometer. The particles decay before their wave functions can overlap, so any interference between amplitudes from the two sources must involve the decay products. For this interference to occur, the wave functions must retain amplitudes for all possible decay channels and angular distributions long after the decay takes place.

As Fig. 1 shows, the system involves electromagnetically induced vector meson emission from relativistic heavy ions at large impact parameter, $\vec{b}$. The electromagnetic field of one nucleus fluctuates to a virtual quark-anti-quark pair which elastically scatters from the other nucleus, emerging as a vector meson [5]. Either nucleus can emit a vector meson. Because neither nucleus is excited in the process, it is impossible to determine which nucleus emitted the photon and which is the target, so the amplitudes add and the system is a two-source interferometer.

Vector meson production may be studied at the recently completed Relativistic Heavy 
Ion Collider (RHIC) at Brookhaven National Laboratory, where gold ions collide at a center of mass energies of 130 to $200 \mathrm{GeV}$ per nucleon. Starting in 2006, the Large Hadron Collider (LHC) at CERN will collide lead ions at a center of mass energy of $5.5 \mathrm{TeV}$ per nucleon. Both accelerators can also study lighter ions.

The production amplitudes may be calculated in the Glauber model [5], with the photon spectrum given by the Weizsäcker-Williams virtual photon method [6]. The nuclear part of the calculation may be compared with experimental data on vector meson photoproduction on nuclear targets; it is accurate within $10-20 \%$. The cross sections are large, about $10 \%$ of the total hadronic cross section at RHIC, rising to $50 \%$ at the LHC [7].

The typical impact parameters for these interaction are large compared with the size of the nuclei. For $\rho$ and $\omega$ meson production, the median impact parameter $\langle b\rangle$ is about 40 fermi at RHIC, rising to 300 fermi at the LHC. For heavier mesons, $\langle b\rangle$ is smaller. These separations are much larger than the nuclear radii, $R_{A} \sim 7 \mathrm{fm}$ for gold or lead. This fact will significantly simplify the calculations. The median impact parameters are also much larger than the lifetimes of the light vector mesons, $c \tau=1.3 \mathrm{fm}$ for the $\rho$ and $c \tau=23 \mathrm{fm}$ for the $\omega$. The vector mesons thus decay before the wave functions can overlap. So, any interference must involve the wave functions after the decay occurs. The final state wave functions must include amplitudes for all of the possible branching ratios and final state angular distributions.

The amplitude $A_{T}$ for observing the vector meson with momentum $\vec{p}$ at a given position in space, $\vec{r}$, and time, $t$, is integrated over the source distribution

$$
A_{T}(\vec{p}, \vec{r}, t)=\int A\left(\vec{p}, \vec{r}, t ; \vec{x}, t^{\prime}\right) d \vec{x} d t^{\prime}
$$

where the local production amplitude $A\left(\vec{p}, \vec{r}, t ; \vec{x}, t^{\prime}\right)$ depends on the electromagnetic field, $E\left(\vec{x}, t^{\prime}\right)$, and the nuclear density, $\rho\left(\vec{x}, t^{\prime}\right)$. The electromagnetic field at a distance $b$ from a relativistic nucleus is a Lorentz-contracted pulse with a width $b / \gamma$, with frequency components extending up to $\hbar \gamma / b[6]$. This pulse may be converted to a photon amplitude at an energy $k$ by a Fourier transform. 
Assuming that the observer is distant, so a vector meson with mass $m_{V}$ and energy $\omega=\sqrt{M_{V}^{2}+|\vec{p}|^{2}}$ may be modelled with a plane wave, and neglecting, for the moment, vector meson decays,

$$
A\left(\vec{p}, \vec{r}, t ; \vec{x}, t^{\prime}\right)=f(\vec{p}, \vec{k}) \rho\left(\vec{x}, t^{\prime}\right) E\left(\vec{x}, t^{\prime}\right) e^{i\left(\vec{p} \cdot(\vec{r}-\vec{x})-\omega\left(t-t^{\prime}\right)\right)}
$$

where the coupling $f(\vec{p}, \vec{k})$ is the amplitude for the electromagnetic field to fluctuate to a quark-antiquark with energy $\vec{k}$ and scatter off the nucleus, emerging as a vector meson with momentum $\vec{p}$. The photon energy spectrum depends on the distance from the emitting nucleus; this is reflected in $E\left(\vec{x}, t^{\prime}\right)$. The required $k_{z}$ depends on $\vec{p}$, with the transverse component $k_{T}$ determined by the the equivalent photon approximation [8]. Absorption of the nascent $\rho^{0}$ is neglected here, but could be included with an additional position-dependent variable. The effect would be the same as modifying $\rho\left(x, t^{\prime}\right)$.

To simplify the calculations, we choose the origin of the coordinate system to be midway between the nuclei at their distance of closest approach. In the center of mass (laboratory) frame, the density is then symmetric (positive parity), while the electric field is antisymmetric (negative parity): $\rho\left(\vec{x}, t^{\prime}\right)=\rho\left(-\vec{x}, t^{\prime}\right)$ and $E\left(\vec{x}, t^{\prime}\right)=-E\left(-\vec{x}, t^{\prime}\right)$. So, the integral in Eq. (1) can be restricted to a single nucleus:

$$
A_{T}(\vec{p}, \vec{r}, t)=\int_{y>0} d \vec{x} d t^{\prime} \rho\left(\vec{x}, t^{\prime}\right) E\left(\vec{x}, t^{\prime}\right) e^{i\left(\vec{p} \cdot \vec{r}-\omega\left(t-t^{\prime}\right)\right)}\left[f(\vec{p}, \vec{k}) e^{i \vec{p} \cdot \vec{x}}-f(\vec{p},-\vec{k}) e^{-i \vec{p} \cdot \vec{x}}\right]
$$

The only differences between the two nuclei are the phases $\pm i \vec{p} \cdot \vec{x}$ and between $f(\vec{p},-\vec{k})$ and $f(\vec{p}, \vec{k})$. The latter is because the sign of $p_{z}$ reduces the symmetry of the system.

The interference and the single-nucleus scattering can be separated by writing $\vec{x}=$ $\vec{b} / 2+\overrightarrow{x^{\prime}}$. The bulk of the cross section occurs when the photon couples coherently to the target nucleus, i.e. when $\vec{k} \cdot \overrightarrow{x^{\prime}} \ll \hbar$. In the rest frame of the target nucleus, the momentum transfer from the target to the vector meson is less than $\hbar / R_{A}$. In the lab frame, this corresponds to transverse and longitudinal limits of $\hbar / R_{A}$ and $\gamma \hbar / R_{A}$ where $\gamma$ is the Lorentz boost. Emitted photons are subject to similar limits, for similar reasons. Because the momentum transfers are so similar, it isn't usually possible to determine which nucleus emitted the photon, and 
which was the scatterer; in fact, at $\vec{p}=0$, the two momentum transfers are equal and opposite. The nuclear form factor can be used to accurately calculate the $\vec{p}$ dependence of the produced mesons.

The coherence is slightly more involved than with real photons, because of the length of the photon pulse. The electromagnetic pulse, with width $|b| / \gamma$, strikes a Lorentz contracted nucleus, with width $R_{A} / \gamma$. These pulses overlap in a region $|z|<b / \gamma$, corresponding to an maximum $\left|p_{z}\right|$ (for full coherence) of the order of $\hbar \gamma / 2 b$. This is tighter than the usual limit that the phase be constant over the target nucleus, $\left|p_{z}\right|<\hbar \gamma / R_{A}$. The difference is because the virtual photon field is relatively poorly localized in $z$. Coherence is maintained over the phase space where interference is significant.

The time dependence of the production, in the exponential and in $E\left(\vec{x}, t^{\prime}\right)$, is symmetric with respect to the two nuclei and factors out into the overall amplitude. The pulse lasts long enough so that $\omega b / 2 c \gamma>\hbar$, and production is not completely coherent over the emission time. This factor is not included in previous calculations. Because there is a pairwise cancellation between space-time volume elements $d \vec{x} d t^{\prime}$ from the two nuclei, the interference is not affected.

With this, $\vec{p} \cdot \vec{x}=\vec{p} \cdot \vec{b} / 2$ and

$$
A_{T}(\vec{p}, \vec{r}, t)=\int_{y>0} d \overrightarrow{x^{\prime}} d t^{\prime} \rho\left(\overrightarrow{x^{\prime}}, t^{\prime}\right) E\left(\overrightarrow{x^{\prime}}, t^{\prime}\right) e^{i\left(\vec{p} \cdot \vec{r}-\omega\left(t-t^{\prime}\right)\right)}\left[f(\vec{p}, \vec{k}) e^{i \vec{p} \cdot \vec{b} / 2}-f(\vec{p},-\vec{k}) e^{-i \vec{p} \cdot \vec{b} / 2}\right] .
$$

We now introduce a few approximations. The amplitude for production from the first nucleus is $A_{1}(\vec{p}, \vec{r}, t)=\int_{y>0} d \vec{x} d t^{\prime} \rho\left(\vec{x}, t^{\prime}\right) E\left(\vec{x}, t^{\prime}\right) f(\vec{p}, \vec{k})$ and

$$
c\left(p_{z}\right)=\frac{\int_{y>0} d \vec{x} d t^{\prime} \rho\left(\vec{x}, t^{\prime}\right) E\left(\vec{x}, t^{\prime}\right) f(\vec{p},-\vec{k})}{\int_{y>0} d \vec{x} d t^{\prime} \rho\left(\vec{x}, t^{\prime}\right) E\left(\vec{x}, t^{\prime}\right) f(\vec{p}, \vec{k})} .
$$

As long as the ratio $f(\vec{p},-\vec{k}) / f(\vec{p}, \vec{k})$ does not vary significantly over the nucleus, the single nucleus production amplitude factors out. The transverse momenta $p_{T}$ and $k_{T}$ do not affect $c$, and $k_{z}$ is determined by $p_{z}$ and $M_{V}$. So,

$$
A_{T}(\vec{p}, \vec{r}, t)=A_{1}(\vec{p}, \vec{r}, t)\left[e^{i \vec{p} \cdot \vec{b} / 2}-c\left(p_{z}\right) e^{-i \vec{p} \cdot \vec{b} / 2}\right]
$$


The amplitude factorizes into an overall amplitude and an interference term. The $p_{T}$ dependence of $A_{1}(\vec{p}, \vec{r}, t)$ is dominated by the nuclear form factors, with the bulk of the production having $p_{T}<2 \hbar / R_{A}$. Most of the uncertainties discussed earlier do not affect the interference term. The time and $z$ variation in $E\left(\vec{x}, t^{\prime}\right)$ should be largely independent of $k$. In the soft Pomeron model, the photon to vector meson coupling increases only slowly with $k$ and has an almost constant phase. At RHIC energies, a photon-meson term is also present, but the phase of $c$ still changes only slowly with $k[5]$.

The interference is clearest when $p_{z}=0$. Then $c=1$ and

$$
A_{T}(\vec{p}, \vec{r}, t)=2 i A_{0} \sin (\vec{p} \cdot \vec{b} / 2)
$$

Here, the approximations involved in defining $c$ disappear. The cross section is then

$$
\sigma \sim\left|A_{T}(\vec{p}, \vec{r}, t)\right|^{2}=2 A_{0}^{2}[1-\cos (\vec{p} \cdot \vec{b})]
$$

This formalism is clearly appropriate for emission of stable particles, such as bremsstrahlung photons. Because of the source separation requirement $b>2 R_{A}$ so that the nuclei do not collide, the interference is clearer than for radiation from $p p$ or $e^{-} e^{-}$collisions [9].

For shortlived particles, the situation is much more interesting. A distant observer will see the decay products of the originally produced mesons. Because of the large variety of possible decays, the wave function becomes rather complicated. In the far-field region, where the particles may be described with plane waves, the formula becomes simpler. If, as expected, the wave function contains amplitudes for all possible decay modes, at a time $t$ much longer than the vector meson lifetime $\tau$, the wave function is

$$
\Psi(t)=\int_{0}^{t} \frac{d t_{d}}{2 \tau}\left[e^{i \vec{p} \cdot\left[\vec{x}_{d}+\vec{b} / 2\right]}+c e^{i \vec{p} \cdot\left[\vec{x}_{d}-\vec{b} / 2\right]}\right] e^{-t_{d} / 2 \tau-i \omega t_{d}} \mid \Sigma_{j} \sqrt{B r_{j}} \Psi_{j}>
$$

where $t_{d}$ is the decay time, at displacement $\vec{x}_{d}=\left(\vec{p} / M_{V}\right) t_{d}$ from the production points. Here, $B r_{j}$ are the branching ratios to different final states, and $\Psi_{j}$ the final state of consisting of $k$ particles

$$
\Psi_{j}=\Sigma_{k} e^{i \overrightarrow{p_{k}}\left[\vec{x}_{k}-\overrightarrow{x_{d}}\right]-\omega_{j}\left(t-t_{d}\right)} \mid \Psi_{j k}>\delta\left(\Sigma_{k} \omega_{k}-\omega\right) \delta\left(\Sigma_{k} \vec{p}_{k}-\vec{p}\right)
$$


Here $\vec{x}_{k}$ are the final state particle positions and $\left|\Psi_{j k}\right\rangle$ includes the particle types and decay angular distribution. The $\delta$ functions impose energy and momentum conservation.

Because of two source terms in Eq. (9), the wave functions of the final state particles are entangled; the phase for the amplitudes from the two sources differs by $\exp [i(\vec{p} \cdot \vec{b}+\delta)]$ where $\delta$ is the phase of $c$. Any measurement on one particle can collapse the wave function of the others [10].

The wave function is observed through interaction with an external detector. This detector could accurately measure either the position or momentum of the final state particles. 'Accurately', is compared to the relevant distance $(b)$ or momentum $(\hbar / b)$ scales. By these metrics, current and planned experiments measure momentum accurately, but not position.

The interference pattern, Eq. (8) can be observed by reconstructing the vector meson transverse momenta, $p_{T}$ [11]. Because $b$ is not easily measurable, $\sigma$ must be integrated over all $b$. This will wash out the interference pattern for $p_{T}>\hbar /\langle b\rangle$. Figure 2 shows the $p_{T}$ spectrum expected for $\rho^{0}$ production at RHIC [11]. A large dip occurs for $p_{T}<\hbar /\langle b\rangle$; this dip is a distinctive experimental signature.

Alternately, in a gedanken experiment, one could instrument the collision region with position sensitive detectors. For example, the decay $J / \psi \rightarrow e^{+} e^{-}$releases $1.54 \mathrm{GeV}$ of energy per electron, producing two ultrarelatistic electrons that are back-to-back in the transverse plane. If the individual decay product momenta $\vec{p}_{k}$ are perpendicular to $\vec{b}$ then it is possible to determine which nucleus emitted the vector meson. At $p_{T}=0$, the production point is on the line between the two $\vec{x}_{k}$. This line will intersect one of the ion trajectories. The nonzero meson $p_{T}$ introduces a small uncertainty, but not enough to make the production point uncertain. The typical $J / \psi p_{T}$ of $50 \mathrm{MeV} / \mathrm{c}$ is small compared to the energy released in the decay. For detectors 500 fermi from the collision point, the $p_{T}$ introduces a pointing uncertainty of 16 fermi, less than the $\langle b\rangle \sim 50$ fermi for $J / \psi$ at the LHC.

A possible setup could consist of two equidistant detectors on opposite sides of the production region. These detectors could randomly measure either position or momentum, like those used for existing tests of Bell's inequality. As with correlated photons, any 
single-detector measurement would be insensitive to the choice of measurement in the other detector. However, when both detectors measured position, the measurements could be combined to determine the production point, localizing the production. Two momentum measurements could be used to reconstruct the interference, demonstrating delocalized production. The only way to maintain both possibilities is if the wave function collapses only when a measurement is actually made. For detectors on opposites sides of a $J / \psi \rightarrow e^{+} e^{-}$ decay, the wave function collapse would have to be superluminal for the correlations to be observable. This is another test of the EPR paradox.

However, there is a broader issue. Most vector mesons have multiple decay modes. For example, the $J / \psi$ can decay to $e^{+} e^{-}, \mu^{+} \mu^{-}, \pi^{+} \pi^{-} \pi^{0}$ or a host of other possibilities. Because $\tau \ll|b| / c$, for interference to occur, the wave function must retain amplitudes for all possible modes long after the decay occurs. This also holds for the final state angular distributions. Otherwise, no interference is possible unless actual information about the decay propagates superluminally. In addition, Eq. (9) shows that the two sources have amplitudes for decaying at a range of different times. The final state wave function must also include amplitudes for all possible decay times. The effect of 'fixing' the decay time before observing the system would be subtler, but could be discerned via detailed studies with positional and momentum detectors.

Quantitative limits on the wave function collapse could be obtained by fitting the measured $p_{T}$ spectrum to a linear combination of coherent (interfering) and incoherent (noninterfering) spectra, $d N / d p_{T}=\zeta d N / d p_{T}(\operatorname{Int})+(1-\zeta) d N / d p_{T}(N I)$, where $\zeta$ is the decoherence parameter and $I$ and $N I$ refer to the interferening and non-interfering components respectively [12]. Because of the continuous variables, this decoherence differs from other studies involving $\Phi \rightarrow K^{0} K^{0}$ or $\Upsilon \rightarrow B \bar{B}[14]$. Also, the presence of two sources complicates the definition of simultaneous production and detection [13]; we work solely in the center of mass frame, which should provide for consistency.

One possible source of reduced coherence, again in a gedanken experiment, involves timing. The vector mesons are produced nearly at rest, but the decay products may be 
relativistic, especially for decays like $J / \psi \rightarrow e^{+} e^{-}$. The maximum flight time difference from the two sources to a detector is $b / c$. If the detectors could resolve times comparable to this difference, then timing could sometimes localize the production point, reducing $\zeta$.

Since the probability of producing a vector meson in grazing collisions $\left(b=2 R_{A}\right)$ is high, about $1 \%$ at RHIC and $3 \%$ at the LHC [7], multiple vector meson production is also observable. Multi-meson final states will exhibit more complicated entanglements, with possibly new behavior.

In conclusion, we have described a 2-source interferometer for short lived particles, and showed that it's description requires a non-local wave function. The observation of interference will be a clear demonstration that, after a decay, a systems' wave function includes amplitudes for all possible decay modes and angular distributions, and does not collapse to a specific decay mode until the wave function is externally observed. Measurements of this interference should be available soon. The STAR detector observed exclusive $\rho^{0}$ production last summer [15]. Next year's data should provide higher statistics data and an accurate $\rho^{0}$ $p_{T}$ spectrum, probing the EPR paradox for continuous variables.

This work was supported by the US DOE, under contract DE-AC-03-76SF00098. 


\section{REFERENCES}

[1] A. Einstein, B. Podolsky and N. Rosen, Phys. Rev. 47, 777 (1935).

[2] J.S. Bell, Physics 1, 195 (1965).

[3] A. Apostolakis et al., Phys. Lett. B422, 339 (1998).

[4] A. S. Parkins and H. J. Kimble, Phys. Rev. A61, 052104 (2000).

[5] T. H. Bauer et al., Rev. Mod. Phys. 50, 261 (1978).

[6] J.D. Jackson, Electrodynamics, 2nd edition, Wiley, 1975.

[7] S. Klein and J. Nystrand, Phys. Rev. C60, 014903 (1999).

[8] M. Vidovic, M. Greiner, C. Best and G. Soff, Phys. Rev. C47, 2308 (1993).

[9] J. Katzenstein, Phys. Rev. 78, 161 (1950).

[10] M. A. Horne, A. Shimony and A. Zeilinger, Phys. Rev. Lett. 62, 2209 (1989).

[11] S. Klein and J. Nystrand, Phys. Rev. Lett. 84, 2330 (2000).

[12] R. A. Berlmann, W. Grimus and B. C. Hiesmayr, Phys. Rev. D60, 114032 (1999).

[13] B. Kayser and L. Stodolsky, Phys. Lett. B359, 343 (1995).

[14] A. Go et al., Phys. Lett. B422, 339 (1998).

[15] S. Klein, nucl-ex/0104016, presented at the 17th Winter Workshop on Nuclear Dynamics, and to appear in the proceedings. 


\section{FIGURES}

FIG. 1. Diagram showing ultra-peripheral vector meson production in heavy ion collisions. The nuclear momenta follow the $z$ axis, and come closest at $z=0$, when their separation (impact parameter), $\vec{b}$ follows the $y$ axis.

FIG. 2. Perpendicular momentum spectra for $\rho^{0}$ production at RHIC, at $p_{z}=0$, for gold on gold collisions at a center of mass energy of $200 \mathrm{GeV}$ per nucleon. Plotted are $d N / d p_{T}$, with and without interference. The curves are normalized to 1 for $p_{T}=0$ and no interference. The calculation assumes that the impact parameter is not measured, so the interference is washed out, except for $p_{T}<25 \mathrm{MeV} / \mathrm{c}$. 